\title{
Characteristic periods of the paleosecular variation of the Earth's magnetic field during the Holocene from global paleoreconstructions
}

\author{
A. González-López ${ }^{\text {a,b, }}$, S.A. Campuzano ${ }^{\mathrm{b}}$, A. Molina-Cardín ${ }^{\text {a,b }}$, F.J. Pavón-Carrasco ${ }^{\text {a }}$, \\ A. De Santis ${ }^{c}$, M.L. Osete ${ }^{a, b}$ \\ ${ }^{a}$ Dpto. Física de la Tierra y Astrofísica, Facultad de Ciencias Físicas, Universidad Complutense de Madrid (UCM), Avd. Complutense s/n, 28040 Madrid, Spain \\ ${ }^{\mathrm{b}}$ Instituto de Geociencias, IGEO (CSIC - UCM), C/ Severo Ochoa 7, Edificio Entrepabellones 7 y 8, 28040 Madrid, Spain \\ ${ }^{\mathrm{c}}$ Istituto Nazionale di Geofisica e Vulcanologia (INGV), Via di Vigna Murata, 605, 00143 Rome, Italy
}

\section{A R T I C L E I N F O}

\section{Keywords:}

Geomagnetic field

Global reconstruction of the geomagnetic field

Frequency analysis

Paleosecular variation

\begin{abstract}
A B S T R A C T
The knowledge of the secular variation of the geomagnetic field at different time scales is important to determine the mechanisms that maintain the geomagnetic field and can help to establish constraints in dynamo theories. We have focused our study on the secular variation at millennial and centennial time scale searching for characteristic periods during the last $10 \mathrm{kyr}$. The frequency study was performed using four recent updated global paleomagnetic field reconstructions (SHA.DIF.14k, CALS10k.2, BIGMUDI4k and SHAWQ2k) by applying three techniques commonly used in signal analysis: the Fourier transform, the Empirical Mode Decomposition, and the wavelet analysis.

Short-term variability of the geomagnetic field energy shows recurrent periods of around 2000, 1000-1400, and $600-800$ and $250-400$ years. The characteristic time around 600-800 years is well determined in all paleomagnetic reconstructions and it is mostly related to the axial dipole and axial octupole terms, but also observable in the equatorial dipole. In addition to this period, longer characteristic times of around 1000-1400 years are found particularly in the equatorial dipole and quadrupole terms in SHA.DIF.14k, CALS10k.2 and BIGMUDI4k while the 2000 year period is only well determined in the total geomagnetic field energy of SHA. DIF.14k and CALS10k.2. The most detailed paleoreconstructions for younger times also detect shortest characteristic times of around 250-400 years.

The long-term variation of the geomagnetic energy is only observable in the axial dipole. A characteristic period of around 7000 years in both SHA.DIF.14k and CALS10k.2 has been found. This long period is related to two decays in the dipole field and a period of increasing intensity. The oldest decay took place between $7000 \mathrm{BCE}$ and 4500 BCE and the present decay that started around 100 BCE. We have modeled the 4500 BCE up to present variation as a combination of a continuous decay, representing the diffusion term of the geomagnetic field, and one pulse that reinforces the strength of the field. Results show a characteristic diffusion time of around 11,000-15,000 years, which is compatible with the diffusion times of the dipole field used in geodynamo theories.
\end{abstract}

\section{Introduction}

One of the most important characteristics of the Earth's magnetic field is its time variability. The changes related to the external contributions of the field include the fastest ones, ranging from seconds to days, and a longer period related to the 11-year cycle of solar activity. The internal contribution driven by the Earth's outer core, also named main geomagnetic field, shows a broad spectrum of frequency time variability (e.g. Constable and Constable 2004): from geomagnetic jerks, with characteristics period of around 6 years, up to reversals, with a mean reversal rate of around 500,000 years for the last $160 \mathrm{Ma}$ (Constable and Korte 2006). The decadal and centennial variability of the main field is known as the secular variation. The analysis of time variability of the geomagnetic field brings constraints on geodynamo

\footnotetext{
* Corresponding author at: Dpto. Física de la Tierra y Astrofísica, Facultad de Ciencias Físicas, Universidad Complutense de Madrid (UCM), Avd. Complutense s/n, 28040 Madrid, Spain.

E-mail address: aliciaglopez@ucm.es (A. González-López).
} 
models (see e.g. Dormy et al. 2000; Terra-Nova et al. 2016).

During the last centuries, the study of the secular variation has been based on magnetic information coming from several sources. For the most recent times, geomagnetic information is provided by geomagnetic observatories at ground and satellite missions (instrumental period). For the historical period, since the 16th - 17th centuries, directional geomagnetic information (declination and inclination) is available thanks mainly to the measurements carried out on board ships during expeditions (see e.g. Jonkers et al. 2003). However, it is not until 19th century, when the first direct measurements of the absolute magnetic field intensity were made by Carl Friedrich Gauss (Gauss 1833). From that epoch to present day, a decreasing trend of the axial dipole field of about $16 \mathrm{nT}$ /year has been observed (Finlay et al. 2016). Prior to the direct measurements, changes in the full geomagnetic field vector are known by making use of archeomagnetism and paleomagnetism.

Information to recover the past geomagnetic secular variation, the paleosecular variation (PSV), is provided from heated archeological artifacts, volcanic rocks or sedimentary records. The most extended record of the PSV is based on lacustrine or marine sediment cores, which are widespread around the globe. However, sedimentary records present some limitations and problems related to the acquisition of the magnetization mechanism. This mechanism, depositional or postdepositional, could cause inclination errors in the remanent magnetization (Anson and Kodama 1987; Tarduno 1990; Tauxe 1993; Roberts et al. 2013, and references therein) and smoothing effects on the PSV due to the progressive and delayed lock-in of the remanence. Moreover, sedimentary records can only provide relative paleointensities.

Absolute paleointensity information is obtained from volcanic rocks or heated archeological artifacts (e.g. kilns). The remanent magnetization in these cases is acquired by a TRM process, well-known when the TRM is carried by single domain particles, thanks to Néel's theory (Néel 1955). Different methodologies are used for paleointensity determination, being one of the most broadly used that based on the original or derived Thellier technique (Thellier and Thellier 1959). Data based on TRM records provide spot values of the past geomagnetic field during the last heating-cooling cycle of the material under study, and therefore, are able to report higher variability than sedimentary materials. However, volcanic rocks are only found in very specific places (i.e. in volcanic regions) and most archeological sites paleomagnetically investigated are generally situated on the Northern Hemisphere, which causes misinformation of the Southern Hemisphere. Besides, the number of archeomagnetic data is low especially before the last 3000 years, so even though they are considered the best way to know the magnetic field variability in the past, the time coverage is short compared to sedimentary records. The paleomagnetic information to investigate the PSV of the Earth's magnetic field is stored in databases, such as GEOMAGIA50.v3 (Brown et al. 2015a, 2015b) or ArcheoInt (Genevey et al. 2008).

The temporal variability of the PSV was investigated by Creer et al. (1990) by using Fourier Transform analysis over a series of directions and relative paleointensities obtained from Lac du Bouchet (France) sediments that spanned the last $100 \mathrm{kyr}$. They found peaks around 10 kyr in the intensity of the field and other ones related to Milankovitch cycles. The relationship between the magnetic field and the orbital parameters of the Earth has also been studied by several authors that analyzed sediment cores spanning millions of years (e.g. Yokoyama and Yamazaki 2000; Yamazaki and Oda 2002; Roberts et al. 2003; Thouveny et al. 2008). More recently, Nilsson et al. (2011) and Panovska et al. (2013) analyzed the secular variation over the Holocene making use of sediment magnetic records distributed around the world. Nilsson et al. (2011) identified a $1350 \mathrm{yr}$ cycle in the dipole tilt for the last $9 \mathrm{kyr}$ from United States, Finland, Japan, Australia and Argentina sediment records. On the contrary, Panovska et al. (2013) found no evidence for globally periodic signals from nearly 50 sediment records. The two aforementioned studies focused on searching for periodicities in paleodirections but not in the paleointensity of the geomagnetic field. On shorter timescales, Roberts et al. (2007) and Jackson and Mound (2010) analyzed observatory data obtaining periodicities on decadal time scales, that seem to be related to changes in the length of the day and with the solar cycle. Moreover, the variability of the PSV can be applied to study other Earth phenomena, such as the radionuclide production rates (Usoskin et al. 2016, and reference therein). In this context, PavónCarrasco et al. (2018) found characteristic times of around 6000 and $800 \mathrm{yr}$ in the radionuclide production rates from two global paleomagnetic reconstructions of the geomagnetic field covering the last 10 kyr.

The most effective approach to study the time variability of the PSV at global scale is to use paleomagnetic reconstructions. Several paleomagnetic reconstructions are now available (e.g., Korte and Constable 2003, 2005; Korte et al. 2009, 2011; Licht et al. 2013; Nilsson et al. 2014; Pavón-Carrasco et al. 2014; Constable et al. 2016; Panovska et al. 2018; Arneitz et al. 2019; Campuzano et al. 2019; Osete et al. 2020). Although there are some differences in the mathematical methodology used to determine the paleoreconstructions, the main differences are related to the use of different input databases. In some of these reconstructions, all the available paleomagnetic information is included, that is, sediment, volcanic and archeomagnetic data (e.g. Constable et al. 2016), while others exclude the sedimentary data (e.g. Pavón-Carrasco et al. 2014). Others include also historical data for the last centuries (e. g., Arneitz et al. 2019) and others apply quality selection criteria based not only on statistical parameters but also on the laboratory protocol used to estimate the paleointensities (Campuzano et al. 2019; Osete et al. 2020).

In this work, we focus our study on investigating the variability of the PSV during the last $10 \mathrm{kyr}$, covering almost the entire Holocene. Our main goal is to verify the presence of characteristic times or periodicities of the geomagnetic field during the Holocene using different global paleomagnetic reconstructions instead of using local sediment cores as the previous works aforementioned. To this scope, we have selected four global paleomagnetic reconstructions: SHA.DIF.14k (Pavón-Carrasco et al. 2014), CALS10k.2 (Constable et al. 2016), BIGMUDI4k (Arneitz et al. 2019) and SHAWQ2k (Campuzano et al. 2019).

The selected global paleomagnetic reconstructions are chosen because they are the most up-to-date ones that cover the Holocene. The SHA.DIF.14k (Pavón-Carrasco et al. 2014) provides the geomagnetic field for the last $14 \mathrm{kyr}$ using archeomagnetic and volcanic data. Sediment data are also included in the CALS10k.2 (Constable et al. 2016) that expands for the last $10 \mathrm{kyr}$, presenting a greater smoothing of the geomagnetic variations, but also better spatial coverage. The SHAWQ2k reconstruction (Campuzano et al. 2019) includes volcanic and archeomagnetic data for the last 2000 years and implements a weighting scheme based on the quality of the laboratory protocol used to obtain the paleomagnetic information, which affects mostly paleointensity data. These three models are developed using Spherical Harmonic Analysis in space and penalized cubic B-splines in time, with knot points every 50 , 40 and 25 years in SHA.DIF.14k, CALS10k.2 and SHAWQ2k, respectively. On the other hand, BIGMUDI4k (Arneitz et al. 2019) is developed by using a Bayesian approach following the methodology described by Leonhardt and Fabian (2007) with a temporal evolution based on cubic B-splines (knot points every $\sim 27$ years). This reconstruction for the last 4000 years uses archeomagnetic and volcanic data (a weighting scheme based on their uncertainties were applied to them), and historical data from the 16th century coming from HISTMAG database (Arneitz et al. 2017). In addition to these Holocene models, the GGF100k reconstruction (Panovska et al. 2018), which covers the last $100 \mathrm{kyr}$, is also analyzed to investigate whether the long period observed in the Holocene is maintained for earlier times.

This paper is organized as follows: Section 2 concerns the used data and the applied analyses; Section 3 shows the frequency analysis results and Sections 4 and 5 present some discussion and conclusions, respectively. 


\section{Data and methodology}

We have used the time series of Gauss coefficients provided by the four selected paleoreconstructions previously described. We have analyzed them individually or some combination of them in order to study the frequency variability between (1) the energy of the different degrees of the harmonic expansion of the geomagnetic field and (2) the axial and equatorial dipoles.

Fig. 1 represents the energy of the total geomagnetic field at the Earth's surface, together with its error band, for the four paleoreconstructions. Total energy is calculated summing up the decomposition of the spatial power spectra at each epoch for each degree, Eq. (1) (Lowes 1974), up to maximum degree $n=5$. Higher degrees than 5, even though modeled by the selected paleoreconstructions, do not have the necessary resolution for the frequency analysis we performed here (see Licht et al. 2013 for more information about the resolution of the models depending on the maximum degree considered).

$R_{n}=(n+1) \sum_{m=0}^{n}\left(g_{n}^{m}\right)^{2}+\left(h_{n}^{m}\right)^{2}$

The error band has been estimated using a Monte - Carlo method to calculate 5000 perturbed sets of Gauss coefficients by randomly selecting values from a normal distribution with mean and standard deviation equal to the Gauss coefficient values and uncertainties, respectively. Then, Eq. (1) is applied for each perturbed set of Gauss coefficients. This procedure allows us to determine an error band at $1 \sigma$ probability for each paleoreconstruction, except for CALS10k.2, because uncertainties are not available in the original publication.

As can be seen in Fig. 1, there exist some differences, but the long trend remains similar: a minimum value in the geomagnetic field energy is observed around 5000-4000 BCE in SHA.DIF.14k and CALS10k.2 which is followed by an increase until 500 BCE - 0 CE. From that time forward, the four geomagnetic reconstructions record a decreasing trend of the geomagnetic field energy which continues until present times (although dotted with highs and lows).

Although Fig. 1 shows the field energy up to harmonic degree 5, we mostly focused our study on changes in the dipole field $(n=1)$ and on the quadrupole and octupole contributions ( $n=2$ and 3 , respectively). The dipole field represents around $90 \%$ of the field at the Earth's surface, while contributions associated with quadrupole or octupole fields could be sufficiently relevant in terms of characteristic times, at least for the last 3000 years (see e.g. Licht et al. 2013). Higher harmonic degrees in the mathematical development of the geomagnetic field derived by paleomagnetic reconstructions may not be reliable enough for this kind

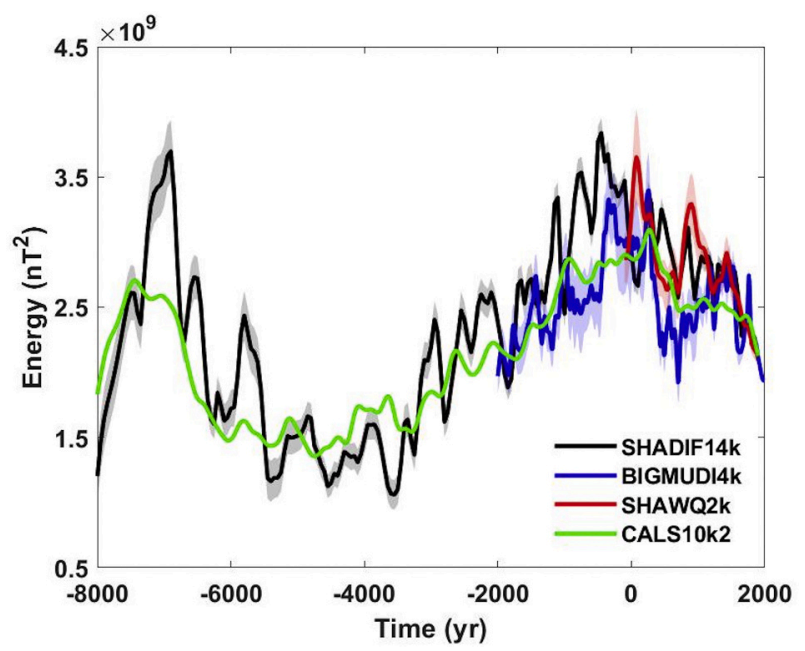

Fig. 1. Total geomagnetic field energy (harmonic degree $n$ from 1 to 5 ) and its standard deviation at the Earth's surface for the four paleomagnetic reconstructions used (see legend inside). of frequency analysis.

In order to identify the dominant frequencies, we firstly carried out a discrete Fourier transform with the periodogram power spectral density estimation (PSD) on the energy of the geomagnetic field shown in Fig. 1. We standardized the time signals, i.e. we subtracted the mean value and divided by its standard deviation. Then, we calculated the PSD, which estimates the power distribution into the discrete frequencies that compose the signal analyzed, allowing us to identify the dominant frequencies in each paleoreconstruction.

Secondly, we applied the Empirical Mode Decomposition (EMD) over the geomagnetic energy using the software developed by Flandrin (2009) for Matlab ${ }^{\circledR}$. The EMD method decomposes a time series into several signals known as Intrinsic Mode Functions (IMFs) and a residual signal. The IMFs are characterized by having zero-mean and fewer oscillation modes than the original signal. The number of IMFs obtained is different in each paleoreconstruction, since it depends on the variability of the geomagnetic field in each model. In order to obtain an estimate of the periodicity of these IMFs we have used one of the methods proposed by Roberts et al. (2007) that applies the Autocorrelation Function (ACF): the IMF is autocorrelated for a range of time delays and if the periodicity exists, there will be peaks that exceed the 95\% confidence level. Those peaks can be related to the periodicity, P: first negative minimum will occur at $\mathrm{P} / 2$, first positive maximum at $\mathrm{P}$, second negative minimum at $3 \mathrm{P} / 2$, and so on.

In order to give robust results we have taken also into account the field energy uncertainty (shown as the error bands in Fig. 1) by using the previously explained Monte - Carlo approach (applied to both techniques, Fourier Transform and EMD). From the perturbed Gauss coefficient sets, we calculate 1000 curves that are analyzed individually providing an ensemble of frequency peaks in the periodogram or IMFs in the EMD. Evaluating the mean of the 1000 results and the standard deviation, we obtain the results with an error band at $1 \sigma$ probability.

Last, we applied the continuous wavelet transformation which is a very useful tool that indicates how the characteristic frequencies of the signal are distributed during the studied time window. Plotting the scalogram is the most common way to analyze wavelets. The scalogram represents the energy density function and is plotted as a contour plot where the horizontal axis is the time and the vertical axis is the frequency or periodicity. We estimate the Cone of Influence (COI), which is the region of the scalogram where results are reliable at $95 \%$ confidence level. Outside the COI, border effects may become important. In the figures included in this work, the region outside the COI will appear as a shaded area and black stripes will be included for periods that cannot be well resolved in order to homogenize the y axis of the figures between paleoreconstructions. These representations show in a very easy way the persistence of characteristic periods over a concrete time interval. In this work, we have used the Wavelet Toolbox of Matlab ${ }^{\circledR}$ considering the Morlet wavelet transform for the representation.

This methodology is very similar to that applied by Panovska et al. (2013).

In addition, based on Fourier Transform and EMD results, we were able to separate the variations of the geomagnetic field energy into 'long-term' and 'short-term' time signals. This decomposition is very important for applying the wavelet analysis because the long-term has more energy than the short-term. The latter would have been masked by the first one if the decomposition had not been carried out. For the longest paleoreconstructions, SHA.DIF.14k and CALS10k.2, which cover the whole time interval of $10 \mathrm{kyr}$, the long-term variations corresponds to the IMF with the largest period plus the residual, while the sum of the other IMFs constitute the short-term variations. Obviously, shorter paleoreconstructions, i.e. BIGMUDI4k and SHAWQ2k, are not able to reproduce the long-term for the Holocene as SHA.DIF.14k and CALS10k.2. Therefore, their short-term variation is estimated as the sum of all their IMFs. In advance, these new time signals will be referred as 'long-term' and 'short-term', for the long-term variations and short-term variations of the geomagnetic field, respectively, omitting the term 
'variations'.

\section{Results}

\subsection{Total field (up to harmonic degree $n=5$ )}

Fig. 2a shows the normalized power spectrum obtained by the Fourier Transform applied over the standardized total field energy calculated from the Eq. (1) for each paleomagnetic reconstruction. The highest peaks in the power spectrum are the ones related to the lowest frequencies (i.e. the largest periods): on SHA.DIF.14k the first peak corresponds to $6400 \mathrm{yr}\left(\sim 1.56 \cdot 10^{-4} \mathrm{yr}^{-1}\right)$; the second one, smaller than the first peak is around $3200-3400 \mathrm{yr}\left(\sim 3.13 \cdot 10^{4} \mathrm{yr}^{-1}\right)$, which agrees with the occurrence of the most remarkable peak on CALS10k.2. For BIGMUDI4k there are two equally powered peaks at $\sim 2600$ and $\sim 1300$ yr $\left(\sim 3.91 \cdot 10^{-4} \mathrm{yr}^{-1}\right.$ and $\sim 7.81 \cdot 10^{-4} \mathrm{yr}^{-1}$, respectively $)$, and SHAWQ2k first peak is around $\sim 1050 \mathrm{yr}\left(\sim 9.37 \cdot 10^{-4} \mathrm{yr}^{-1}\right)$, which is observed also in SHA.DIF.14 k and CALS10k.2 but with smaller power spectrum. These two models show common minor peaks at $\sim 1600 \mathrm{yr}$ $\left(6.25 \cdot 10^{-4} \mathrm{yr}^{-1}\right)$ while a peak at $\sim 2100 \mathrm{yr}\left(4.69 \cdot 10^{-4} \mathrm{yr}^{-1}\right)$ is only observed in SHA.DIF.14 k. Relevant peaks corresponding to periods lower than $1000 \mathrm{yr}$ are found in the range between 600 and $800 \mathrm{yr}$ $\left(1.70 \cdot 10^{-3}-1.25 \cdot 10^{-3} \mathrm{yr}^{-1}\right)$ in the four models. For CALS10k.2 the last important peak that can be observed is $\sim 540 \mathrm{yr}\left(1.86 \cdot 10^{-3} \mathrm{yr}^{-1}\right)$, meanwhile for SHA.DIF. $14 \mathrm{k}$ is $\sim 440 \mathrm{yr}\left(2.27 \cdot 10^{-3} \mathrm{yr}^{-1}\right)$. Periods in that range are observed to be more prominent in BIGMUDI4k and SHAWQ2k, especially the common period in the range of 200-300 yr (0.005-0.003 $\mathrm{yr}^{-1}$ ). These two paleomagnetic reconstructions continue detecting closer minor peaks beyond $0.01 \mathrm{yr}^{-1}$ (100 yr), however, these could be related to the separation of the selected knot points during the modeling approach. Thus, we decided to cut the horizontal axis at this value.

Results applying the EMD to the total magnetic field energy are shown in Fig. 2b. Each IMF period obtained by EMD has an associated error band at $1 \sigma$ probability. Results are in agreement with Fourier analysis within uncertainties.

Obtained results are compatible with the temporal window studied in each global model and the input database used. The CALS10k.2 reconstruction includes sedimentary records that smooth the variability of the geomagnetic field, which favors the detection of long-term variations but prevents those of short-term. On the other hand, BIGMUDI4k and SHAWQ2k, that span a shorter time interval, are able to model quicker variations of the geomagnetic field allowing to detect shorter

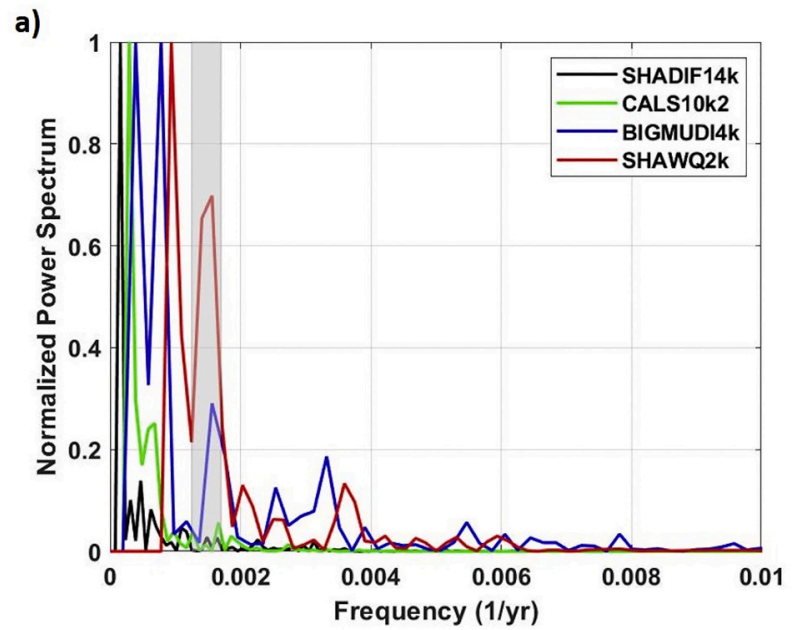

periods but cannot record the longer ones. From Fig. 2b one can see clearly a gap between periods of around 2000 years and nearly 7000 years (only found in the longest paleoreconstructions). This marks the boundary between short-term (lower IMFs periods) and long-term (higher IMFs periods) of the geomagnetic field for the Holocene.

Finally, the wavelet analysis of the total field energy is shown in Fig. 3. We have set the same color scale limits for each paleoreconstruction for a better comparison, although this means the saturation or the smoothing in some plots.

For SHA.DIF.14k and CALS10k. 2 the most powerful (higher magnitude value) period is related to the long-term, with a period above 5000 yr. It is statistically significant only from 4000 to 2000 BCE in both reconstructions, but it is observed over the total time interval. Another characteristic period present in both reconstructions is observed around $1500 \mathrm{yr}$, shown in light blue (magnitude $10^{8}-1.3 \cdot 10^{8}$ ) for the CALS10k.2, which seems to be present in BIGMUDI4k as its most remarkable period. Also, a common period of around $600-800 \mathrm{yr}$ is observed in the four reconstructions. It is more remarkable in SHAWQ2k, but also important in the SHA.DIF.14k and BIGMUDI4k. This period is not so well-observed in CALS10k.2. Other remarkable common short periods is around 250-400 yrs., which is observed in SHA.DIF.14k (more intense between 3000 and 0 CE), in BIGMUDI4k and SHAWQ2k between $0 \mathrm{CE}$ and $1000 \mathrm{CE}$.

\subsection{Dipole field (harmonic degree $n=1$ )}

For the dipole energy, results are very similar to the ones presented in Section 3.1 for the first 5 harmonic degrees, since the dipole field represents nearly the $90 \%$ of the main total field at the Earth's surface. Fourier transform results as well as characteristic periods of the IMFs are summarized in Table S1 in Section 1 of Supplementary Material and Fig. S1 shows the wavelet analysis.

As in Section 3.1, the periods with the highest magnitude in the dipole energy of SHA.DIF.14k and CALS10k.2 correspond to the longterm (Fig. S1a and S1b). In order to highlight the short-term of the dipole field, we made the decomposition into short- and long-term following the results of the EMD procedure. From Fig. $2 \mathrm{~b}$ it is clear that the short-term is characterized by periods lower than $4000-5000 \mathrm{yr}$. Thus, for SHA.DIF.14k and CALS10k.2, the longest paleoreconstructions, the short-term is defined by the sum of all the IMFs except the largest one with values around 7000 yr. For BIGMUDI4k and SHAWQ2k all the IMFs represent the short-term signal (see Fig. 2b),

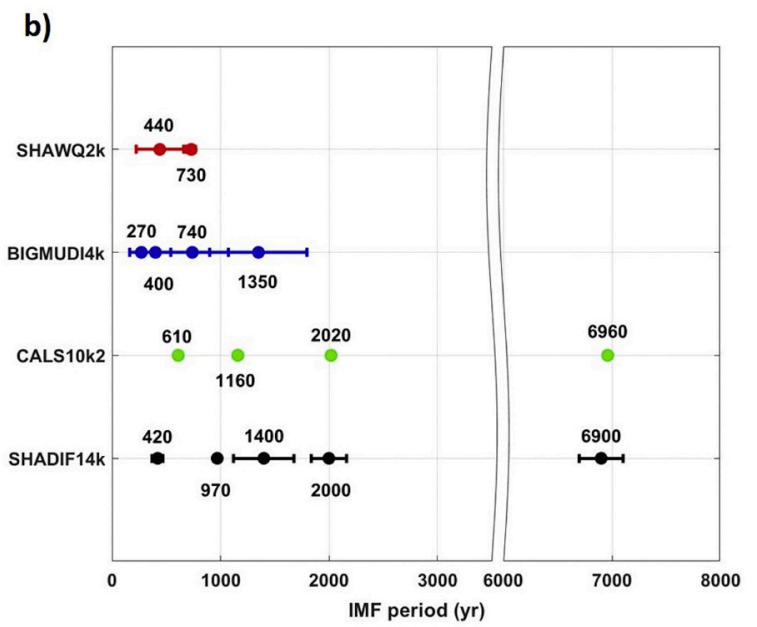

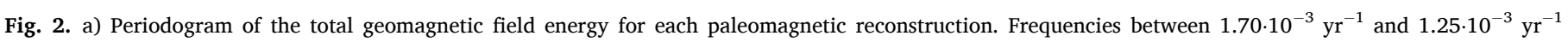

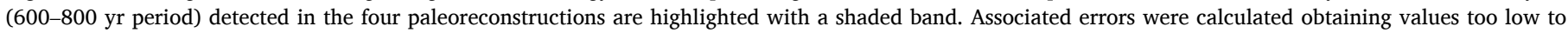

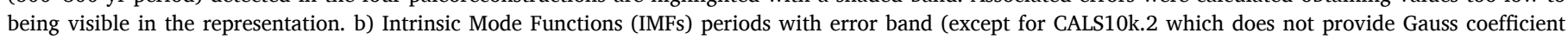

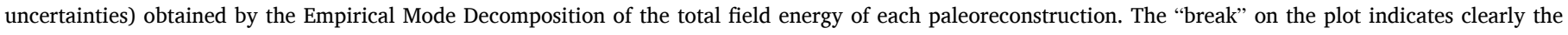
separation between long- and short-term of the geomagnetic field for the last $10 \mathrm{kyr}$. 


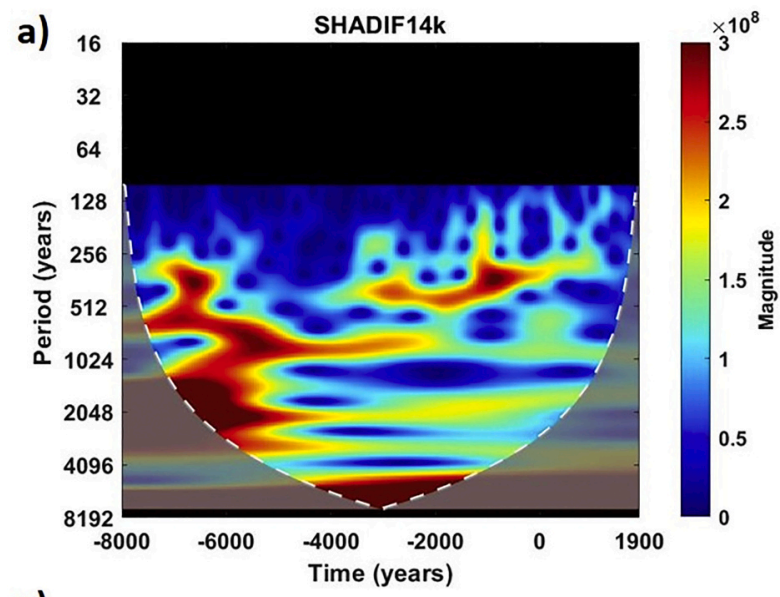

c)

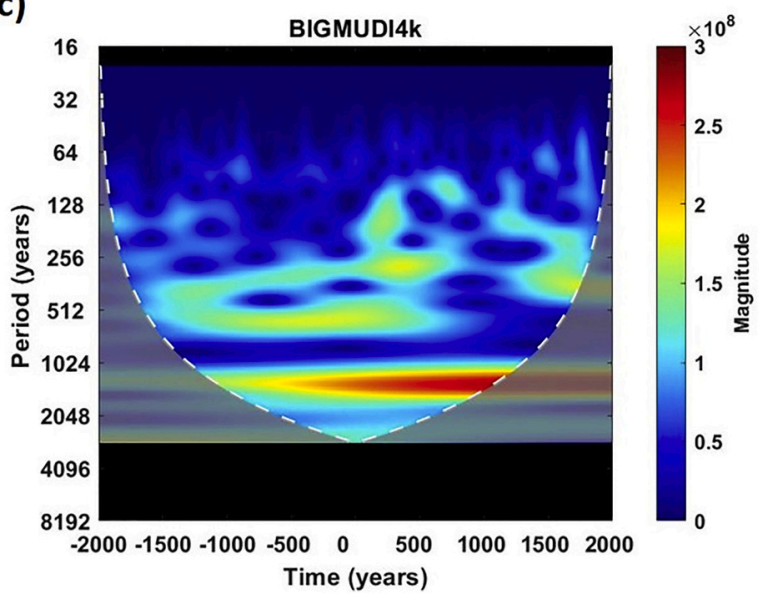

b)

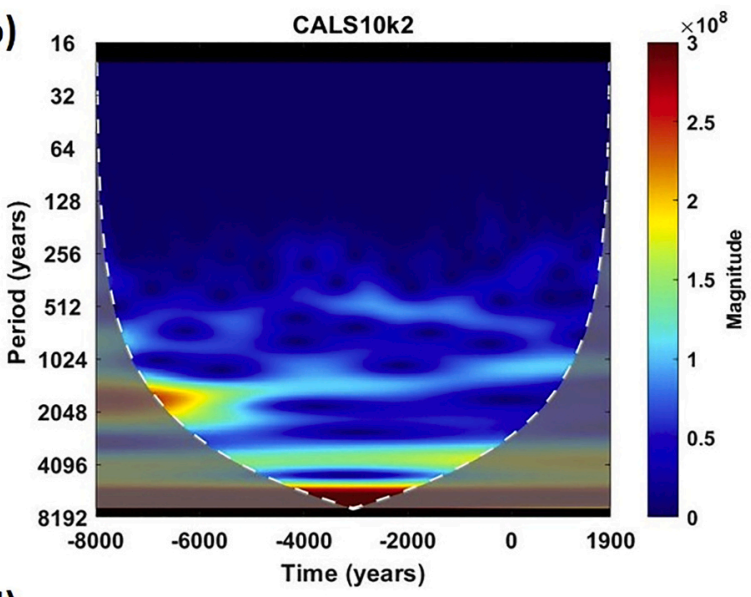

d)

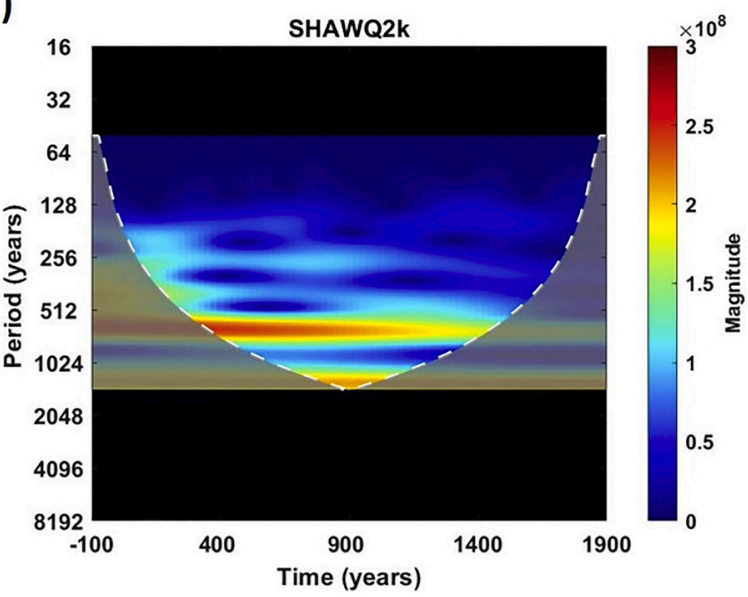

Fig. 3. Wavelet analysis of the total geomagnetic field energy (up to $n=5$ ) for: a) SHA.DIF.14k; b) CALS10k.2; c) BIGMUDI4k; and d) SHAWQ2k.

therefore the sum of all of them defines the short-term.

A characteristic time around $600-800 \mathrm{yr}$ is found in all paleoreconstructions (Fig. 4) when the long-term signal is filtered. In addition to this period, characteristic times between 1300 and $2000 \mathrm{yr}$ are also found for SHA.DIF.14 k, CALS10k.2 and BIGMUDI4k. The shortest characteristic times, 250-400 yr, are detected in BIGMUDI4k and SHAWQ2k reconstructions. A more detailed analysis of Fig. 4 is reported in Section 1 of Supplementary Material.

In order to know which Gauss coefficient dominates the above observed frequencies of the dipole energy, we have also analyzed separately the first three Gauss coefficients.

A quick comparison between the time evolution of the first three Gauss coefficients for the last $10 \mathrm{kyr}$ provides valuable information about the long trend (Fig. 5a). We can see clearly that long-term is only present on the axial dipole (represented as the absolute value of the first Gauss coefficient, $g_{1}^{0}$, for better comparison with the total and dipole energy) of both SHA.DIF.14k and CALS10k.2. The EMD approach applied to $\left|g_{1}^{0}\right|$ indicates that the long trend can be represented by the longest IMF period plus the residual showing characteristic periods of $7050 \pm 260 \mathrm{yr}$ for SHA.DIF. 14k and $6860 \mathrm{yr}$ for CALS1 0k. 2 (see Table S2 in Supplementary Material). Note again that to describe the long trend we use only these two geomagnetic reconstructions because they cover the whole studied period.

Differences observed in Fig. $5 \mathrm{~b}$ can be attributed to the input database since CALS10k.2 also includes sediment records. However, an agreement is observed for the last decay that seems to start around 100 BCE - 0 CE, obtaining for SHA.DIF. $14 \mathrm{k}$ a slope of $-2.5 \pm 0.5 \mathrm{nT} / \mathrm{yr}$ and for CALS10k.2 a slope of $-2.7 \pm 0.5 \mathrm{nT} / \mathrm{yr}$.

Since the axial dipole carries the long-term for the last $10 \mathrm{kyr}$ with a characteristic period of around $7000 \mathrm{yr}$, it could be interesting to check if this oscillation is observed for earlier times or it is a particular feature of the Holocene. To investigate this topic we have also analyzed the axial dipole evolution provided by the GGF100k paleoreconstruction (Panovksa et al., 2018) that extends for the last $100 \mathrm{kyr}$. The GGF100k includes the Laschamp excursion ( $\sim 41 \mathrm{kyr} \mathrm{BP}$ ) which is clearly observable in this model (Fig. S4). To avoid this event, where the PSV could not be representative of a stable behavior of the geomagnetic field, we have focused our analysis on the last $30 \mathrm{kyr}$. We have identified 5 axial dipole increases after the Laschamp excursion that seem to occur every 4000-5000 yr (Fig. S4b). According to the EMD (Table S5) and wavelet analysis (Fig. S5) the long-term variations of the axial dipole according to the GGF100k paleoreconstruction seems to exhibit a shorter period of around $5800 \mathrm{yr}$.

Coming back to the short-term dipole evolution for the last $10 \mathrm{kyr}$, we have also compared the wavelet analysis results for the axial and equatorial terms (Fig. 6), the latter obtained by the quadratic sum of the non-axial Gauss coefficients $g_{1}^{1}$ and $h_{1}^{1}$. Results suggest that neither axial nor the equatorial component are the main responsible of the whole short-term of the dipole energy. A 600-800 yr period is common in the four reconstructions for both axial and equatorial terms, being in general more powerful in the axial one. In contrast, the $1300 \mathrm{yr}$ period is more prominent in the equatorial dipole. This could be related to the dipole tilt oscillation observed by Nilsson et al. (2011) during the last 9 kyr. Here, we can ensure its presence during the last $6 \mathrm{kyr}$ with SHA.DIF.14k or more conservative for CALS10k. 2 in the last $5 \mathrm{kyr}$. BIGMUDI4k also presents this period for the last $2 \mathrm{kyr}$. Finally, the $250-400 \mathrm{yr}$ period is also more powerful in the equatorial dipole for SHAWQ2k than in the axial one, and it is present during almost the entire interval.

For detailed information about the frequency decomposition of the axial and equatorial terms, please see Section 1 of the Supplementary 

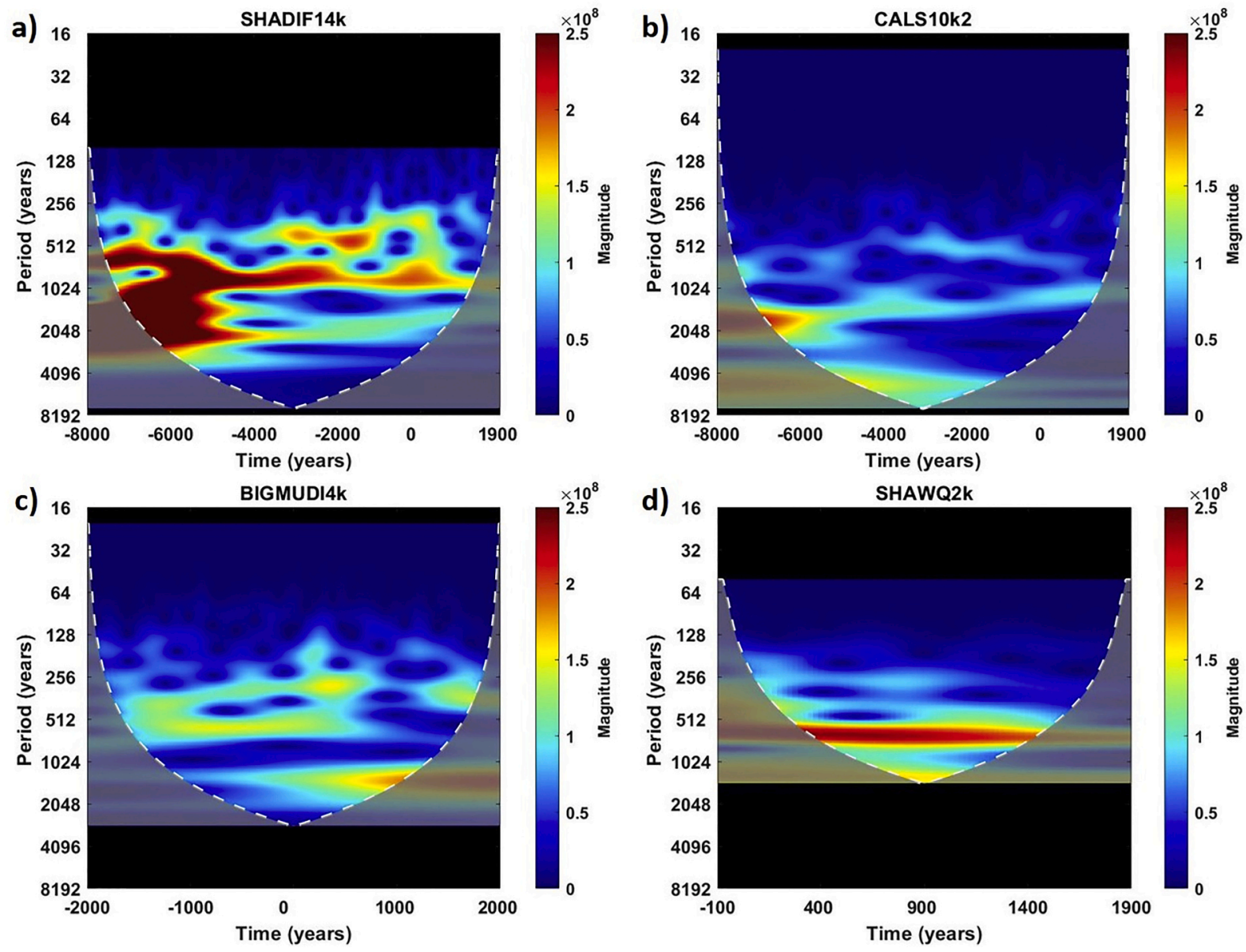

Fig. 4. Wavelet analysis of the short-term of the dipole field for: a) SHA.DIF.14k; b) CALS10k.2; c) BIGMUDI4k; and d) SHAWQ2k.

a)
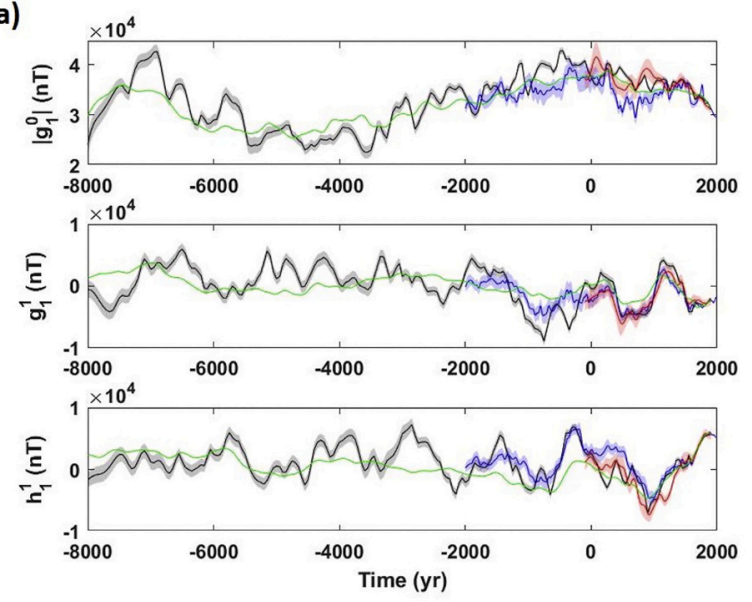

b)

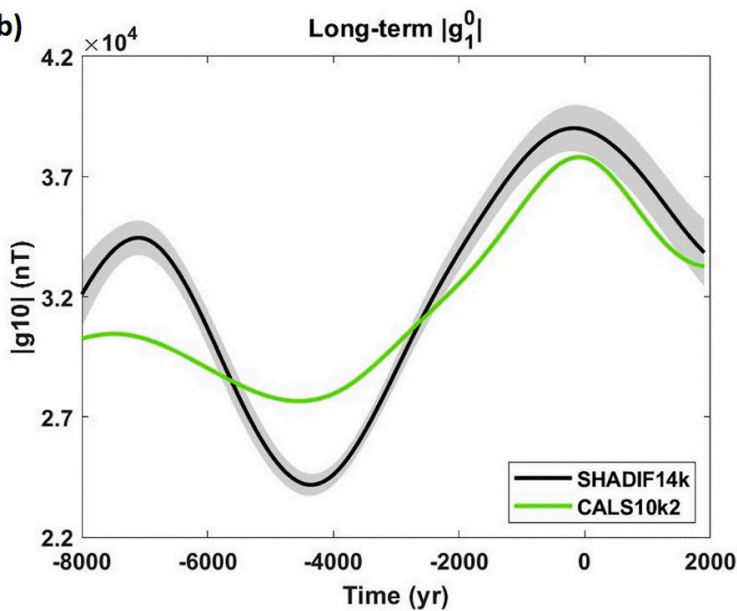

Fig. 5. a) Temporal evolution of the first three Gauss coefficients: absolute value of $g_{1}^{0}$ (top), $g_{1}^{1}$ (middle) and $h_{1}^{1}$ (bottom) for SHA.DIF.14k (black), CALS10k.2 (green), BIGMUDI4k (blue) and SHAWQ2k (red). b) Long-term of the $\left|g_{1}^{0}\right|$ from SHA.DIF.14k (black) with error band and CALS10k.2 (green) without it as it does not provide Gauss coefficient uncertainties. (For interpretation of the references to color in this figure legend, the reader is referred to the web version of this article.)

Material.

3.3. Non-dipole field: Quadrupole (harmonic degree $n=2$ ) and Octupole (harmonic degree $n=3$ ) fields

We focus here on the study on the quadrupole and octupole fields since these terms are the ones that contribute most strongly to the non- dipole field. However, for completeness, a study of the non-dipole field from harmonics degree $n=2$ to $n=5$ can be found in Section 2 of Supplementary Material and Fig. S2 and Fig. S3.

Results from the Fourier analysis and EMD for quadrupole and octupole fields are given in Tables S3 and S4 of Supplementary Material, respectively. Fig. 7 shows the wavelet analysis of the short-term of quadrupole (left) and octupole (right) field energy. 
a)

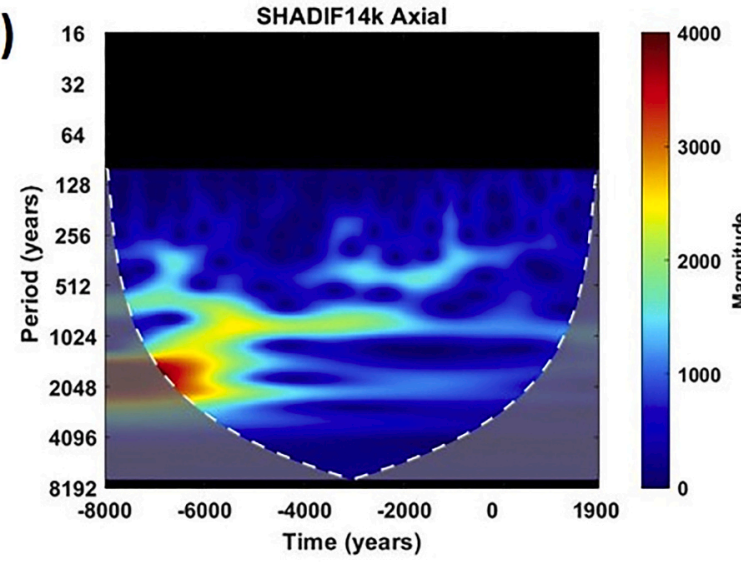

b)

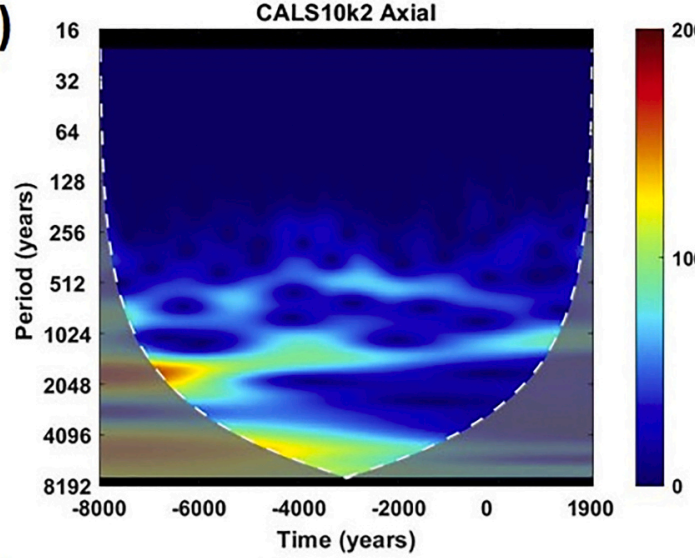

c)

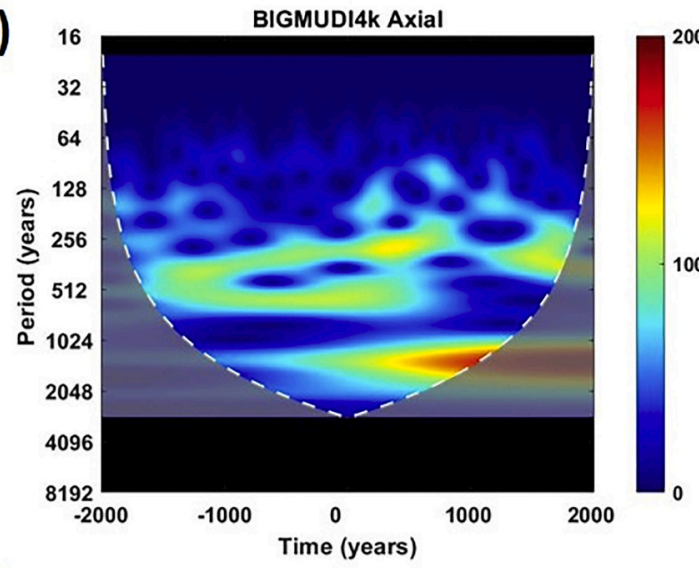

d)

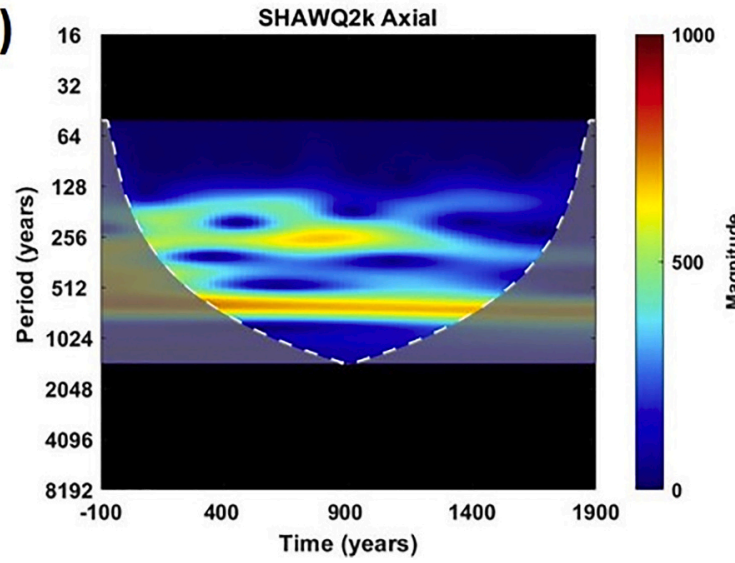

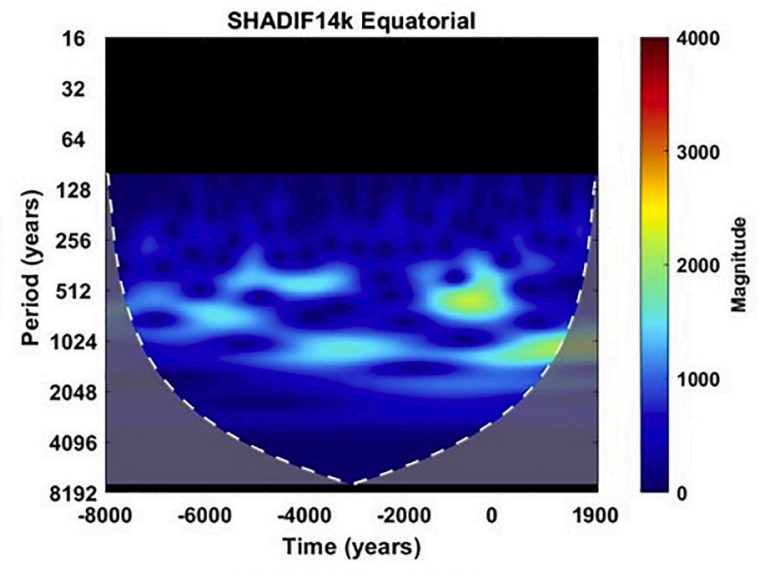
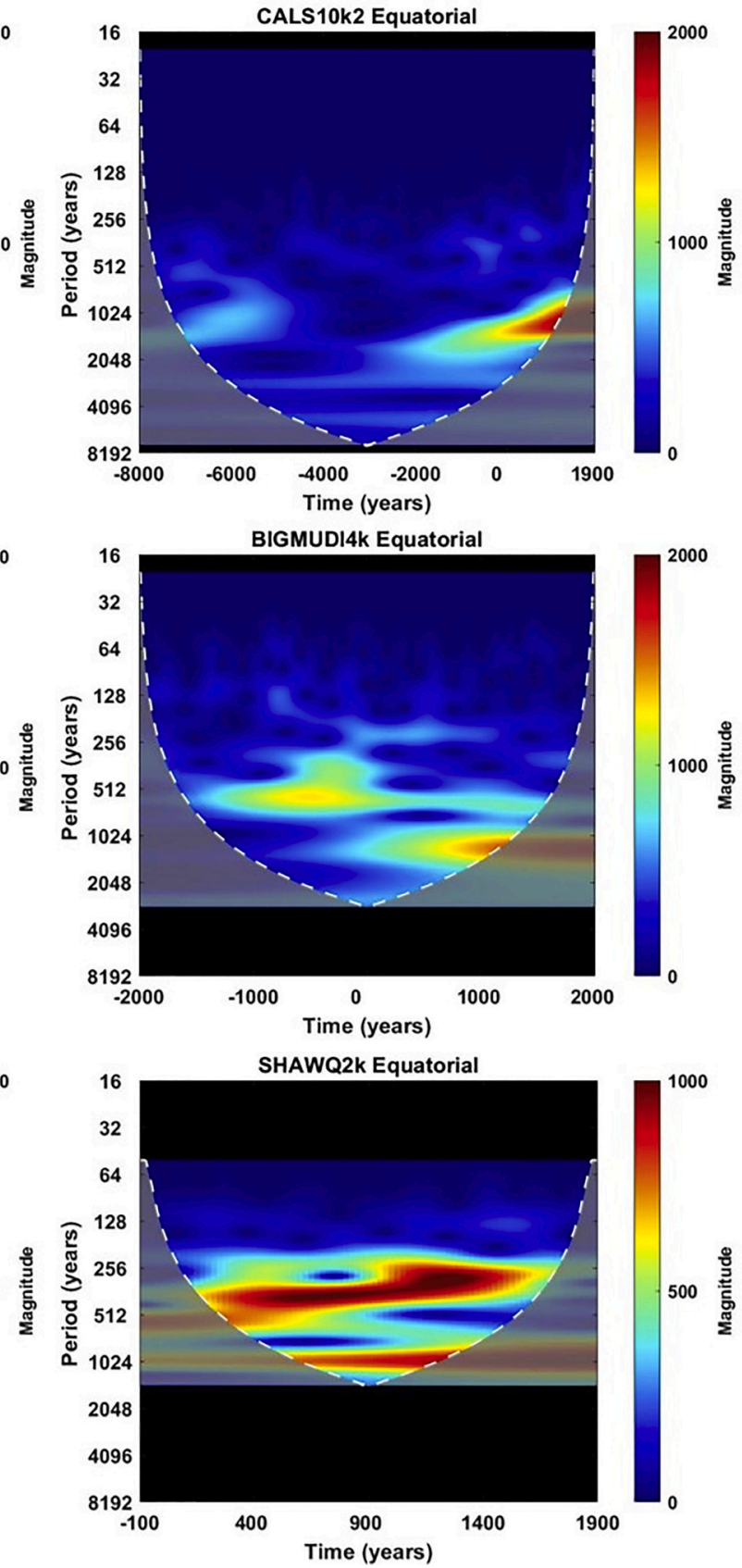

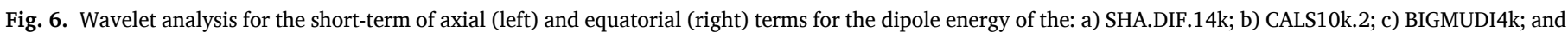
d) SHAWQ2k paleomagnetic reconstructions. Note that the color scale has been unified in each paleomagnetic reconstruction separately but not between them. 

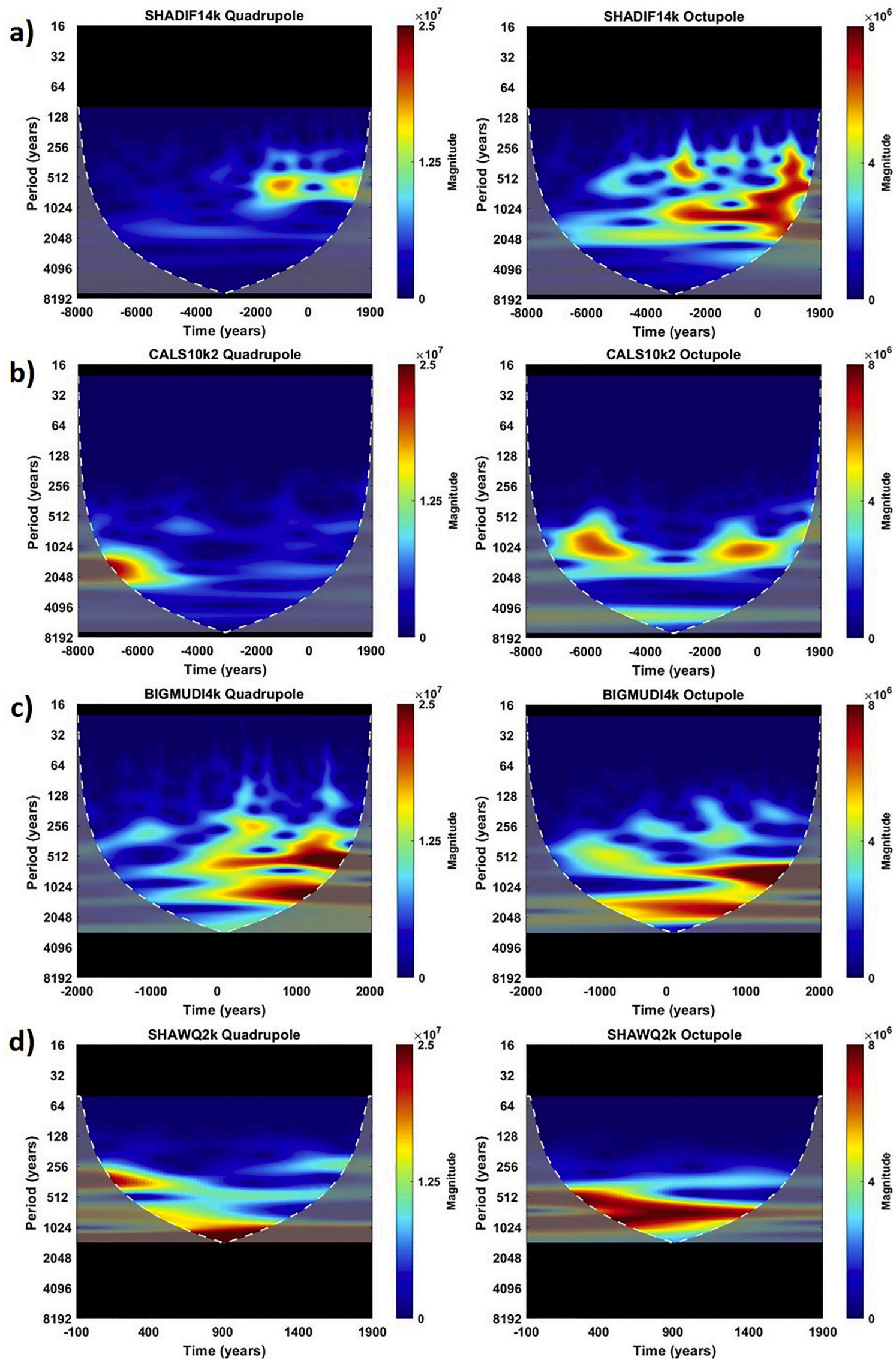

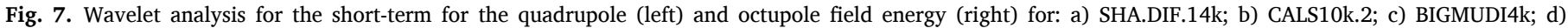
SHAWQ2k. Note that the color scale has been unified for each degree. 
Note that in the SHA.DIF.14k paleoreconstruction, these higher degrees seem to increase its contribution to the variability of the field in the last 4000-6000 years. This could be due to the uneven temporal distribution of the data used in the modeling process (see Pavón-Carrasco et al. 2014) that causes that for the earlier times only the dipole field is well constrained.

As can be seen in Fig. 7, non-dipole terms share some periods with the dipole (Fig. 6). The 600-800 yr and $1200-1500$ yr periods are observed in practically all the four reconstructions, better observed in the octupole contribution. However, the quadrupole field in SHA. DIF.14 $\mathrm{k}$ also shows an intensified period of around 500-700 $\mathrm{yr}$ from 3000 BCE to present day. The $600-800 \mathrm{yr}$ period seems to be absent in the quadrupole contribution of CALS10k.2 and SHAWQ2k.

Table 1 summarizes the characteristic periods obtained by applying the three frequency analysis methodologies to the four paleoreconstructions of the geomagnetic field for different magnetic contributions. We calculated the variance of the original signal (each field contribution studied) and the variance of their IMFs, obtaining the percentage of variance each IMF with approximate characteristic period can explain from the original signal (Peel et al. 2005). These values complement the relevance of each period at different time windows showed by the wavelet analysis (Figs. 3, 4, 6 and 7).

\section{Discussion}

\subsection{Long-term analysis of the axial dipole field}

We evaluated if the long-term oscillation for the last $10 \mathrm{kyr}$ could be reproduced by a combination of a diffusion process continuously affecting, and sporadic pulses that reinforced the strength of the axial dipole field.

We considered the diffusion as a decreasing exponential, Eq. (2), with a characteristic decay time, $\tau$, which can be related to the dipole diffusion times,

$\left|g_{1}^{0}\right|=k \exp \left(-\frac{t}{\tau}\right)$

We followed the approximation described in Chapter 9.1 of Merrill et al. (1998) that considers the induction equation with the advective term equal to 0 . This approximation leads to a simplified equation of the geomagnetic field proportional to a decreasing exponential where the decay time depends on the dimension of the source of the field and the electric conductivity of the outer core. The decay time also depends on the degree of the field expansion, varying for the dipole between $5 \mathrm{kyr}$ and $50 \mathrm{kyr}$ for conductivities between $10^{5} \mathrm{Sm}^{-1}$ and $10^{6} \mathrm{Sm}^{-1}$, respectively (Merrill et al., 1998). Geodynamo models usually refer their results as function of magnetic diffusion times (e.g. Olson et al. 2009), usually around of $200 \mathrm{kyr}$, which is four times the dipole diffusion time, estimated between $30 \mathrm{kyr}$ and $50 \mathrm{kyr}$ (Olson 2015). These values are compatible with the ones predicted by the simplification done by Merrill et al. (1998), even though they used a velocity field equal to 0 which is not considered in the diffusion times of geodynamo models.

Since the long-term of the $\left|g_{1}^{0}\right|$ increases between 4500 BCE and 100 BCE in both paleoreconstructions (Fig. 5b), there should be a mechanism that confronts the decay. We explored a model where this mechanism is a pulse $p(t)$ defined by a skew normal shape (Ashour and Abdel-hameed 2010), Eq. (3), that allows us to evaluate different scenarios depending on its skewness parameter, $\alpha$ (Fig. S6),

$p(t)=A \cdot e^{\frac{-(t-\mu)^{2}}{2 \sigma^{2}}} \cdot\left[1+\operatorname{erf}\left(\frac{\alpha}{\sqrt{2}} \cdot \frac{t-\mu}{\sigma}\right)\right]$,

where erf corresponds to the error function, $A$ is a factor that accounts for the intensity of the pulse in nT/yr, $\mu$ is the time at which the pulse is located and $\sigma$ the scale (width) of the pulse. Note that when $\alpha$ is equal to 0 , the pulse becomes a normal distribution where $\mu$ and $\sigma$ would represent the mean and the standard deviation, respectively.

Thus, we have considered a model where a continuous decay is taking place defined by Eq. (2) but a single skew normal pulse, Eq. (3), also occurs in some time interval between 5000 BCE to present. We did not evaluate the first increase-decay step (between 8000 BCE and 5000 BCE) because it is worse characterized in SHA.DIF.14 k and CALS10k.2. The lack of archeomagnetic data in these times reduces their reliability comparing with more recent times.

Fitting between the model and the observed long-term of the $\left|g_{1}^{0}\right|$ is carried out by randomly sampling parameters space $\left(y_{0}, \tau, A, \mu, \sigma, \alpha\right)$. For each random combination of parameter values, the pulse is calculated and then the corresponding $\left|g_{1}^{0}\right|$ series is built step by step from the initial point, $y_{0}$ (the $\left|g_{1}^{0}\right|$ value at $t=-5000$ ), decreasing its value following the diffusion and then adding the pulse (Fig. S7). More information about the fitting process can be found in Section 4 of Supplementary Material.

First, we chose a broad range in the parameters space that defines the fit allowing $\alpha$ to vary between -60 and 60 which includes skew normal function to the left $(\alpha<0)$ and to the right $(\alpha>0)$. That allowed us to discard right skewed pulses since the sum of squared residuals (SSR) were several orders of magnitude higher than the left skewed pulses. We evaluate a broad range of tests keeping $\alpha$ negative. Finally, the best fit able to explain in both paleoreconstructions the pulse and the decay is found in a range of $\alpha$ between -30 and -10 . The pulse presents a sharp decrease that allows to fit better the decay of $\left|g_{1}^{0}\right|$ from 100 BCE to present-day since the pulse is less relevant during that time interval. The characteristic time decay is between 11,000 and 15,000 $\mathrm{yr}$ in both paleoreconstructions. These best fits along with the observed long-term variations of SHA.DIF.14k and CALS10k.2 are shown in Fig. 8 with the most probable values for the characteristic time decay $(\tau)$ at $95 \%$ probability (shown as pink bar).

Considering only the pulse, it represents the secular variation of the field if the diffusion were not present (Fig. S7b). The greatest rates of increase of the axial dipole field would reach values of around $6-8 \mathrm{nT} / \mathrm{yr}$ at $\sim 1400$ BCE in SHA.DIF.14k and $\sim 800$ BCE in CALS10k.2. Computing the accumulative pulse will allow us to figure out how the shape of $\left|g_{1}^{0}\right|$ would be if only the increasing pulse is present from 5000 BCE to present times (Fig. S8). During that time interval of almost 7000 years, the longterm of $\left|g_{1}^{0}\right|$ would increase between 20,000 nT and 30,000 nT, which is the double of its real increase between its minimum (around $4500 \mathrm{BCE}$ ) and its maximum (around $100 \mathrm{BCE}$ ) with a value of around 10,000 nT and 15,000 nT for CALS10k.2 and SHA.DIF.14k, respectively. Following this approach, this would mean that the diffusion takes nearly the $50 \%$ of the increase provided by the reinforcement mechanism. Table S6 in Section 4 of the Supplementary Material collects the detailed results of the best fit in each paleoreconstruction.

We can also evaluate what would happen if a new pulse were not occur in the next millennia. The diffusion will dominate the long-term of the axial dipole exponentially decaying its intensity within $11,000-15,000$ yr. However, it seems unlikely based on the observation of the last $30 \mathrm{kyr}$ studied with the GGF100k in section 3.1. A next pulse will be expected to occur in the next millennia following the succession of pulses observed every $4000-5000$ yr. However, it could also indicate a continuous decay in the energy of the long-term of the axial dipole that may leads to an excursion, such as the Laschamp event. Our model is limited to the latest increase of the long-term and we are not able to predict the future behavior of the geomagnetic field.

Even though the representation of the reinforcement mechanism as a skewed pulse is a very simplistic idea, it is worth noting that the estimated decay times required for the fit are in the same order of magnitude with the times given in the geodynamo literature (e.g. Merrill et al., 1998; Dormy et al. 2000; Terra-Nova et al. 2016).

\subsection{Short-term analysis}

Within the short-term variability, common characteristic periods are 
Table 1

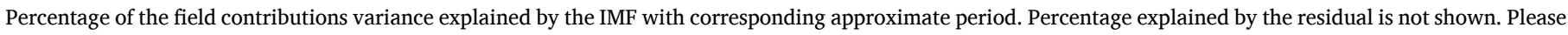

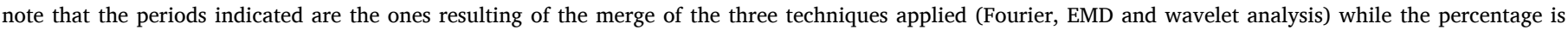
calculated from the IMFs obtained with the EMD.

\begin{tabular}{|c|c|c|c|c|c|c|c|c|c|c|c|}
\hline \multirow{3}{*}{$\begin{array}{l}\text { Field Contribution } \\
\text { Model } \backslash \text { Period } \\
7000\end{array}$} & \multicolumn{4}{|c|}{ Total field $(n=1-5)$} & \multicolumn{4}{|c|}{ Dipole field $(\mathrm{n}=1)$} & \multicolumn{3}{|c|}{ Axial Dipole $\left(\left|g_{1}^{0}\right|\right)$} \\
\hline & SHA,DIF.14k & CALS10k.2 & BIGMUDI4k & SHAWQ2k & SHA,DIF.14k & CALS10k.2 & BIGMUDI4k & SHAWQ2k & SHA,DIF.14k & CALS10k.2 & BIGMUDI4k \\
\hline & 58.5 & 36.8 & & & 15 & 36.8 & & & 58.8 & 29.9 & \\
\hline 2000 & 2.2 & 5.8 & & & 4.3 & 5.8 & 36.6 & & 1.9 & 7.2 & 17.3 \\
\hline $1000-1400$ & 8.6 & 5.3 & 28.9 & & & 5.2 & & & 9.4 & 7.2 & \\
\hline $600-800$ & 3.4 & 1.2 & 11.5 & 27.1 & 8.6 & 1.2 & 17.4 & 31.2 & 3.2 & 1.2 & 16.9 \\
\hline $250-400$ & 1.9 & & 7.6 & 5.8 & & & 4.1 & 5.6 & 1.1 & & 10.1 \\
\hline
\end{tabular}

\begin{tabular}{|c|c|c|c|c|c|c|c|c|c|c|c|c|}
\hline \multirow{2}{*}{$\frac{\text { Axial Dipole }\left(\left|g_{1}^{0}\right|\right)}{\text { SHAWQ2k }}$} & \multicolumn{4}{|c|}{ Equatorial Dipole $\left(\sqrt{ }\left(\left(g_{1}^{1}\right)^{2}+\left(h_{1}^{1}\right)^{2}\right)\right)$} & \multicolumn{4}{|c|}{ Quadrupole field $(n=2)$} & \multicolumn{4}{|c|}{ Octupole Field $(n=3)$} \\
\hline & SHA,DIF.14k & $\begin{array}{l}\text { CALS10k.2 } \\
16.4\end{array}$ & BIGMUDI4k & SHAWQ2k & $\begin{array}{l}\text { SHA,DIF.14k } \\
2.9\end{array}$ & CALS10k.2 & BIGMUDI4k & SHAWQ2k & SHA,DIF.14k & CALS10k.2 & BIGMUDI4k & SHAWQ2k \\
\hline & & 30.1 & & & & 16.1 & & & 1.7 & 9.7 & 15.3 & \\
\hline 23.3 & 23 & 34.1 & 10 & 51 & 2.4 & 37.6 & 7.5 & 68.8 & 7.4 & 39.3 & 10.2 & \\
\hline 44.8 & & & 17.6 & & 2.6 & 4.8 & 4.6 & & 7.1 & 11.8 & & 40.8 \\
\hline 0.8 & 24 & 4.5 & 2.4 & 16.5 & 9 & & 4.6 & 5.6 & 5.5 & & 7.8 & 9.2 \\
\hline
\end{tabular}

found around of 2000, 1000-1400, 600-800 and 250-400 yr when combining the results of the three methodologies applied (see Table 1). More detailed information about the periods identified in the different field contributions can be found in Table S1 - S4 of the Supplementary Material.

The period of around $2000 \mathrm{yr}$ detected in the EMD of SHA.DIF.14k and CALS10k.2 (Fig. 2b) has not a clear representation in the wavelet analysis (Fig. 3a and b) where it cannot be well differentiated from the 1000-1400 yr. It is better defined in the octupole term meanwhile the wavelet analysis of the quadrupole field does not show it in any of the studied reconstructions. Quadrupole field highlights the 1000-1400 yr period which is clearly seen in the total field analysis of SHA.DIF.14k, CALS10k.2 and BIGMUDI4k. In SHAWQ2k we can only assure the presence of a $1000 \mathrm{yr}$ period, which is particularly relevant in its equatorial dipole. This component carries the period of around $1400 \mathrm{yr}$ in all other reconstructions, which may be related to the dipole-tilt of 1350 yr observed by Nilsson et al. (2011).

The period of around $600-800 \mathrm{yr}$ is observed in the total energy of the geomagnetic field being especially significant for dipole and octupole contributions. Since dipole and octupole fields are dominated by the zonal harmonic (order $\mathrm{m}=0$, i.e. $\mathrm{g}_{1}^{0}$ and $\mathrm{g}_{3}^{0}$ ), belonging to the same antisymmetric geodynamo families ( $\mathrm{n}+\mathrm{m}$ odd), then it is intriguing to evaluate if there is any correlation between characteristic periods of Gauss coefficients belonging to the same geodynamo families (Gubbins and Zhang 1993; Merrill et al., 1998). Geodynamo families are a classification proposed firstly by Roberts (1971) based on the symmetry respect to the equator of the $\alpha$-effect (antisymmetric) and $\omega$-effect (symmetric) in the dynamo theory. The Gauss coefficients can be categorized following this separation depending on whether their degree plus order is even (symmetric) or odd (antisymmetric). We evaluated each Gauss coefficient (up to harmonic degree $n=5$ ) by EMD and wavelet analysis, but we find no clear correlation between other coefficients of the same geodynamo family apart from $g_{1}^{0}$ and $g_{3}^{0}$ (see Section 5 of Supplementary Material and Fig. S9 and Fig. S10).

The shortest periods of around 250-400 yr are remarkable in the dipole field of BIGMUDI4k and SHAWQ2k, but they seem to be more prominent in the axial component for BIGMUDI4k while for SHAWQ2k these periods dominate in the equatorial dipole.

\section{Conclusions}

A detailed frequency analysis has been carried out in order to study the characteristic times associated with the PSV of the geomagnetic field during the Holocene using the combination of the results obtained with
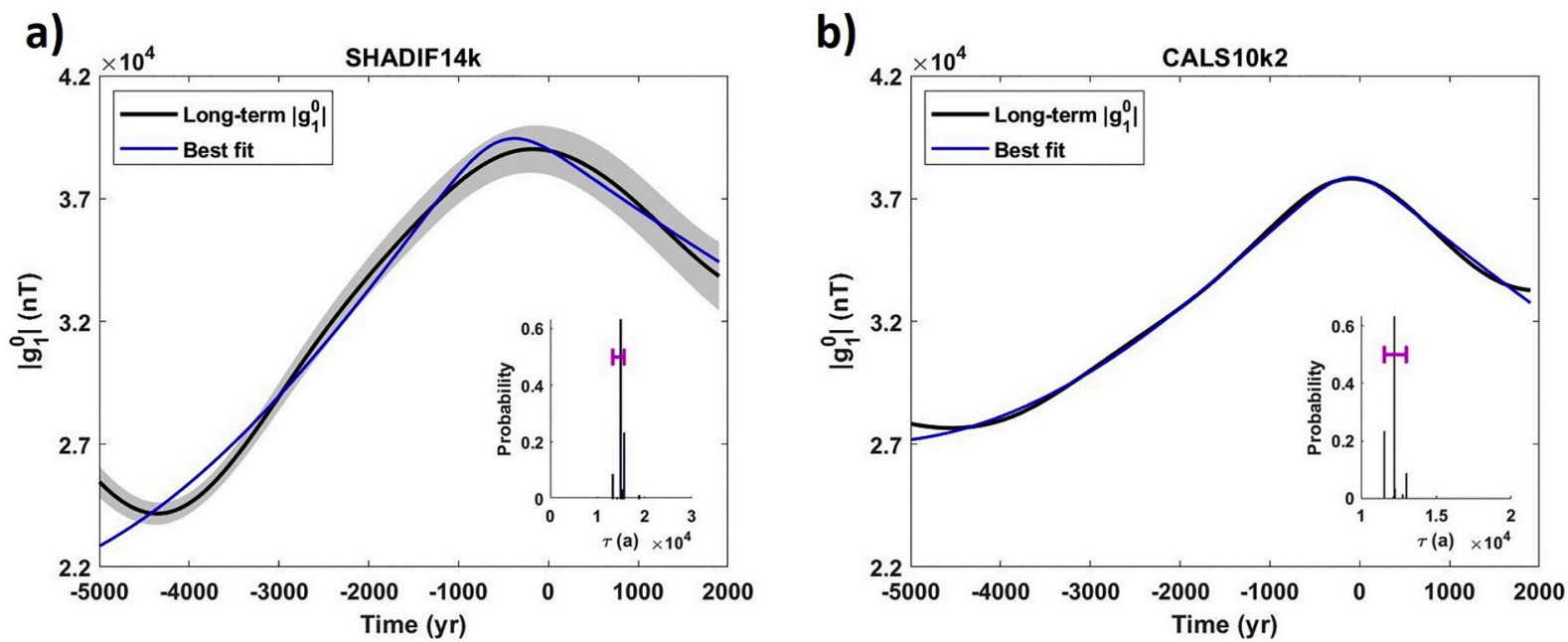

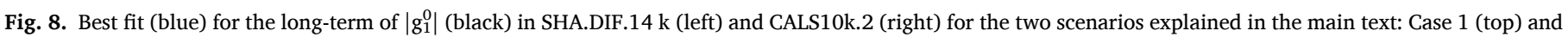

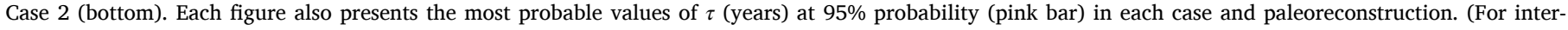
pretation of the references to color in this figure legend, the reader is referred to the web version of this article.) 
three different methodologies. The analysis has been computed over four relevant global paleoreconstructions for the Holocene. Note that discrepancies between models regarding intermittent or not persistent periods can be due to the different input databases and different time covered by each paleoreconstruction. However, all of them present common features, such as a characteristic period around 600-800 yr, and the longest paleoreconstructions (i.e., SHA.DIF.14k and CALS10k.2) detected also periods of 2000 and $1000-1400 \mathrm{yr}$, the last one also observed in BIGMUDI4k. Paleoreconstructions that cover a shorter time window, i.e. BIGMUDI4k and SHAWQ2k, also present a good agreement showing common periods around $250-400$ yr. Frequencies in the total geomagnetic field (up to degree 5) are governed by the frequencies in the dipole field. In particular, the axial dipole seems to be responsible for the $600-800 \mathrm{yr}$ period detected in the dipole field since this period has a higher intensity in axial term than in the equatorial one. Equatorial dipole shows a $1300 \mathrm{yr}$ period in SHA.DIF.14k, CALS10k.2 and BIGMUDI4k. Meanwhile, in SHAWQ2k the most relevant period in the equatorial term is of around $300 \mathrm{yr}$.

The long-term of the geomagnetic field is governed by the axial dipole showing two decreases and only one increase in the last $10 \mathrm{kyr}$. We have explored a model that reproduces the latest increase and decay from a combination of diffusion and reinforcement mechanism. The diffusion was represented by an exponential decrease following the approximation made by Merrill et al. (1998) with characteristic decay times of around 11,000-15,000 yr, which have the same order of magnitude of the diffusion dipole times used in geodynamo theories $(\sim 20 \mathrm{kyr})$. The reinforcement mechanism is considered as a pulse defined by a skewed normal function that allowed us to evaluate a great range of different pulses. The best fits indicate that this pulse would be left skewed which shows a faster rate of decay than the rate of increase. The accumulative pulse between 5000 BCE and present day estimates the real increase of the long-term of $\left|g_{1}^{0}\right|$ if the diffusion mechanism were not present, reaching values of around $20,000-30,000 \mathrm{nT}$, which is the double of the observed growth in the strength of $\left|g_{1}^{0}\right|$ between its latest minimum and maximum (of around 10,000-15,000 nT). This would mean that the diffusion takes nearly the $50 \%$ of the increase provided by the reinforcement mechanism.

Additional efforts should be done to improve the databases prior to 3000 BCE times to better constrain the paleoreconstructions of the geomagnetic field and, hence, to extend the knowledge of the geomagnetic field variability to the past.

\section{Declaration of Competing Interest}

None.

\section{Acknowledgements}

Financial support was given by the Spanish Ministry of Education (FPU17/03635) and Spanish Ministry of Science and Innovation (CGL2017-92285-EXP, CGL2017-87015-P and PGC2018-099103-AI00). SAC thanks to Juan de la Cierva Formación Program (FJC2018037643-I), and AMC acknowledges the contract funding from CGL201787015-P project (FEDER/Ministerio de Ciencia, Innovación y Universidades - Agencia Estatal de Investigación). ADS would like to thank INGV and UCM that cofunded his short stay at UCM, Madrid, for completing the present work. Authors would like to thank the Editor and two anonymous reviewers for their useful comments and suggestions that improve the quality of this manuscript.

\section{Appendix A. Supplementary data}

Supplementary data to this article can be found online at https://doi. org/10.1016/j.pepi.2021.106656.

\section{References}

Anson, G.L., Kodama, K.P., 1987. Compaction-induced inclination shallowing of the postdepositional remanent magnetization in a synthetic sediment. Geophys. J. Roy. Astr. S. 88 (3), 673-692.

Arneitz, P., Leonhardt, R., Schnepp, E., Heilig, B., Mayrhofer, F., Kovacs, P., Hejda, P., Valach, F., Vadasz, G., Hammerl, C., Egli, R., Fabian, K., Kompein, N., 2017. The HISTMAG database: combining historical, archaeomagnetic and volcanic data. Geophys. J. Int. 2010, 1347-1359. https://doi.org/10.1093/gji/ggx245.

Arneitz, P., Egli, R., Leonhardt, R., Fabian, K., 2019. A Bayesian iterative geomagnetic model with universal data input: self-consistent spherical harmonic evolution for the geomagnetic field over the last 4000 years. Phys. Earth Planet. Inter. 290, 57-75. https://doi.org/10.1016/j.pepi.2019.03.008.

Ashour, S.K., Abdel-hameed, M.A., 2010. Approximate skew normal distribution. J. Adv. Res. 1 (4), 341-350. https://doi.org/10.1016/j.jare.2010.06.004.

Brown, M.C., Donadini, F., Korte, M., Nilsson, A., Korhonen, K., Lodge, A., Lengyel, S.N., Constable, C.G., 2015a. GEOMAGIA50.v3: 1. General structure and modifications to the archeological and volcanic database. Earth Planet. Sp. 67, 83. https://doi.org/ 10.1186/s40623-015-0232-0.

Brown, M.C., Donadini, F., Nilsson, A., Panovska, S., Frank, U., Korhonen, K., Schuberth, M., Korte, M., Constable, C.G., 2015b. GEOMAGIA50.v3: 2. A new paleomagnetic database for lake and marine sediments. Earth Planet. Sp 67, 70. https://doi.org/10.1186/s40623-015-0233-z.

Campuzano, S.A., Gómez-Paccard, M., Pavón-Carrasco, F.J., Osete, M.L., 2019. Emergence and evolution of the South Atlantic anomaly revealed by the new paleomagnetic reconstruction SHAWQ2k. Earth Planet. Sc. Lett. 512, 17-26. https:// doi.org/10.1016/j.epsl.2019.01.050.

Constable, C.G., Constable, S.C., 2004. Satellite magnetic field measurements: applications in studying the deep earth. In: Sparks, R., Hawkesworth, C. (Eds.), The State of the Planet: Frontiers and Challenges in Geophysics. https://doi.org/ 10.1029/150GM13.

Constable, C.G., Korte, M., 2006. Is the Earth's magnetic field reversing? Earth Planet. Sc. Lett. 246, 1-16. https://doi.org/10.1016/j.epsl.2006.03.038.

Constable, C., Korte, M., Panovska, S., 2016. Persistent high paleosecular variation activity in southern hemisphere for at least 10000 years. Earth Planet. Sc. Lett. 453, 78-86. https://doi.org/10.1016/j.epsl.2016.08.015.

Creer, K.M., Thouveny, N., Blunk, I., 1990. Climatic and geomagnetic influences on the lac du Bouchet palaeomagnetic SV record through the last 110000 years. Phys. Earth Planet. Inter. 64 (2-4), 314-341. https://doi.org/10.1016/0031-9201(90) 90046-Z.

Dormy, E., Valet, J.-P., Courtillot, V., 2000. Numerical models of the geodynamo and observational constraints. Geochem. Geophys. Geosyst. 1, 1037. https://doi.org/ 10.1029/2000GC000062.

Finlay, C., Aubert, J., Gillet, N., 2016. Gyre-driven decay of the Earth's magnetic dipole. Nat. Commun. 7, 10422. https://doi.org/10.1038/ncomms10422.

Flandrin, P., 2009. Matlab Toolbox: Empirical mode decomposition. http://perso.enslyon.fr/patrick.flandrin/software2.html.

Gauss, C.F., 1833. Die Intensität der erdmagnetischen Kraft, zurückgeführt auf absolutes Maass. Ann. Phys. 104 (6), 241-273. https://doi.org/10.1002/andp.18331040602.

Genevey, A., Gallet, Y., Constable, C., Korte, M., Hulot, G., 2008. ArcheoInt: An upgraded compilation of geomagnetic field intensity data for the past ten millennia and it application to the recovery of the past dipole moment. Geochem. Geophys. Geosyst. 9 (4) https://doi.org/10.1029/2007GC001881.

Gubbins, D., Zhang, K., 1993. Symmetry properties of the dynamo equations for paleomagnetism and geomagnetism. Phys. Earth Planet. Inter. 75, 225-241. https:// doi.org/10.1016/0031-9201(93)90003-R.

Jackson, L., Mound, J., 2010. Geomagnetic variation on decadal time scales: what can we learn from empirical mode decomposition? Geophys. Res. Lett. 37, L14307 https:// doi.org/10.1029/2010GL043455.

Jonkers, A.R.T., Jackson, A., Murray, A., 2003. Four centuries of geomagnetic data from historical records. Rev. Geophys. 41 (2), 1006. https://doi.org/10.1029/ 2002RG000115.

Korte, M., Constable, C.G., 2003. Continuous global geomagnetic field models for the past 3000 years. Phys. Earth Planet. Inter. 140, 73-89. https://doi.org/10.1016/j. pepi.2003.07.013.

Korte, M., Constable, C.G., 2005. Continuous global geomagnetic field models for the past 7 millenia: 2. CALS7k. Geochem. Geophys. Geosyst. 6 https://doi.org/10.1029/ 2004GC000801. Q02H16.

Korte, M., Donadini, F., Constable, C.G., 2009. Geomagnetic field for 0-3ka: 2. A new series of time-varying global models. Geochem. Geophys. Geosyst. 10 https://doi. org/10.1029/2008GC002297. Q06008.

Korte, M., Constable, C., Donadini, F., Holme, R., 2011. Reconstructing the Holocene geomagnetic field. Earth Planet. Sc. Lett. 312 (3-4), 497-505. https://doi.org/ 10.1016/j.epsl.2011.10.031.

Leonhardt, R., Fabian, K., 2007. Paleomagnetic reconstruction of the global geomagnetic field evolution during the Matuyama/Brunhes transition: iterative Bayesian inversion and independent verification. Earth Planet. Sc. Lett. 253 (1-2), 172-195. https://doi.org/10.1016/j.epsl.2006.10.025.

Licht, A., Hulot, G., Gallet, Y., Thébault, E., 2013. Ensembles of low degree archeomagnetic field models for the past three millennia. Phys. Earth Planet. Inter. 224, 38-67. https://doi.org/10.1016/j.pepi.2013.08.007.

Lowes, F.J., 1974. Spatial power Spectrum of the Main geomagnetic field, and extrapolation to the Core. Geophys. J. R. Astron. Soc. 36, 717-730.

Merrill, R.T., McElhinny, M.W., McFadden, P.L., 1998. The Magnetic Field of the Earth: Paleomagnetism, the Core, and the Deep Mantle. Academic Press, San Diego. 
Néel, L., 1955. Some theoretical aspects of rock. Adv. Phys. 4, 191-243. https://doi.org/ 10.1080/00018735500101204.

Nilsson, A., Muscheler, R., Snowball, I., 2011. Millennial scale cyclicity in the geodynamo inferred from a dipole tilt reconstruction. Earth Planet. Sc. Lett. 311, 299-305. https://doi.org/10.1016/j.epsl.2011.09.030.

Nilsson, A., Holme, R., Korte, M., Suttie, N., Hill, M., 2014. Reconstructing Holocene geomagnetic field variation: new methods, models and implications. Geophys. J. Int. 198 (1), 229-248. https://doi.org/10.1093/gji/ggu120.

Olson, P., 2015. 8.01 - Core Dynamics: An Introduction and Overview. In: Treatise on Geophysics (2 ${ }^{\text {nd }}$ Edition). Elsevier, pp. 1-25. https://doi.org/10.1016/B978-0-44453802-4.00137-8.

Olson, P., Driscoll, P., Amit, H., 2009. Dipole collapse and reversal precursors in a numerical dynamo. Phys. Earth Planet. Inter. 173, 121-140. https://doi.org/ 10.1016/j.pepi.2008.11.010.

Osete, M.L., Molina-Cardín, A., Campuzano, S.A., Aguilella-Arzo, G., BarrachinaIbáñez, A., Falomir-Granell, F., Oliver Foix, A., Gómez-Paccard, M., MartínHernández, F., Palencia-Ortas, A., Pavón-Carrasco, F.J., Rivero-Montero, M., 2020. Two archaeomagnetic intensity maxima and rapid directional variation rates during the Early Iron Age observed at Iberian coordinates. Implications on the evolution of the Levantine Iron Age Anomaly. Earth Planet. Sc. Lett. 533, 116047. https://doi. org/10.1016/j.epsl.2019.116047.

Panovska, S., Finlay, C.C., Hirt, A.M., 2013. Observed periodicities and the spectrum of field variations in Holocene magnetic records. Earth Planet. Sc. Lett. 379, 88-94. https://doi.org/10.1016/j.epsl.2013.08.010.

Panovska, S., Constable, C.G., Korte, M., 2018. Extending global continuous geomagnetic field reconstructions on timescales beyond human civilization. Goechem. Geophys. Geosyst. 19, 4757-4772. https://doi.org/10.1029/2018GC007966.

Pavón-Carrasco, F.J., Osete, M.L., Torta, J.M., De Santis, A., 2014. A geomagnetic field model for the Holocene base don archaeomagnetic and lava flow data. Earth Planet. Sc. Lett. 388, 98-109. https://doi.org/10.1016/j.epsl.2013.11.046.

Pavón-Carrasco, F.J., Gómez-Paccard, M., Campuzano, S.A., González-Rouco, J.F., Osete, M.L., 2018. Multi-centennial fluctuations of radionuclide production rates are modulated by the Earth's magnetic field. Sci. Rep. 8, 9820. https://doi.org/10.1038/ s41598-018-28115-4.

Peel, M.C., Amirthanathan, G.E., Pegram, G.G.S., McMahon, T.A., Chiew, F.H.S., 2005. Issues with the application of the empirical mode decomposition analysis. In: Zerger, A., Argent, R.M. (Eds.), MODSIM 2005 International Congress on Modelling and Simulation. Modelling and Simulation Society of Australia and New Zealand, pp. 1682-1687. December 2005. https://www.mssanz.org.au/modsim05/papers /peel.pdf.

Roberts, G.O., 1971. Spatially periodic dynamos. Phil. Trans. R. Soc. London A266, 535-558. https://doi.org/10.1098/rsta.1970.0011.

Roberts, A.P., Winklhofer, M., Liang, W.T., Horng, C.S., 2003. Testing the hypothesis of orbital (eccentricity) influence on Earth's magnetic field. Earth Planet. Sc. Lett. 216 (1-2), 187-192. https://doi.org/10.1016/S0012-821X(03)00480-1.

Roberts, P.H., Yu, Z.J., Russel, C.T., 2007. On the 60-year signal from the core. Geophys. Astrophys. Fluid Dyn. 101, 11-35. https://doi.org/10.1080/03091920601083820.

Roberts, A.P., Tauxe, L., Heslop, D., 2013. Magnetic paleointensity stratigraphy and high-resolution quaternary geochronology: successes and future challenges. Quat. Sci. Rev. 61, 1-16. https://doi.org/10.1016/j.quascirev.2012.10.036.

Tarduno, J.A., 1990. Absolute inclination values from deep sea sediments: a reexamination of the cretaceous Pacific record. Geophys. Res. Lett. 17, 101-104. https://doi.org/10.1029/GL017i001p00101.

Tauxe, L., 1993. Sedimentary records of relative paleointensity: theory and practise. Rev. Geophys. 31, 319-354. https://doi.org/10.1029/93RG01771.

Terra-Nova, F., Amit, H., Hartmann, G.A., Trindade, R.I.F., 2016. Using archaeomagnetism field models to constrain the physics of the core: robustness and preferred locations of reversed flux patches. Geophys. J. Int. 206, 1890-1913. https://doi.org/10.1093/gji/ggw248.

Thellier, E., Thellier, O., 1959. Sur l'intensité du champ magnétique terrestre dans le passé historique et géologique. Ann. Geophys. 15, 285-376.

Thouveny, N., Bourlès, D.L., Saracco, G., Carcaillet, J.T., Bassinot, F., 2008. Paleoclimatic context of geomagnetic dipole lows and excursions in the Brunhes, clue for an orbital influence on the geodynamo? Earth Planet. Sc. Lett. 275, 269-284. https://doi.org/10.1016/j.epsl.2008.08.020.

Usoskin, I.G., Gallet, Y., Lopes, F., Kovaltsov, G.A., Hulot, G., 2016. Solar activity during the Holocene: the Hallstatt cycle and its consequence for grand minima and maxima. Astron. Astrophys. 27295.

Yamazaki, T., Oda, H., 2002. Orbital influence on Earth's magnetic field: 100,000-year periodicity in inclination. Science 295 (5564), 2435-2438. https://doi.org/10.1126/ science. 1068541.

Yokoyama, Y., Yamazaki, T., 2000. Geomagnetic paleointensity variation with a $100 \mathrm{kyr}$ quasi-period. Earth Planet. Sc. Lett. 181 (1-2), 7-14. https://doi.org/10.1016/ S0012-821X(00)00199-0. 